\title{
What's the evidence for fining the unvaccinated?
}

\author{
Cite as: CMAJ 2022 January 31;194:E132-3. doi: 10.1503/cmaj.1095985
}

Posted on cmajnews.com on January 14, 2022

Q uebec's recent move to tax people who refuse COVID-19 vaccinations reopened debate about the merits of such penalties.

Premier François Legault said that choosing to remain unvaccinated has consequences for the health care system and not all Quebecers should foot the bill.

Roughly one in 10 adults in Quebec remain unvaccinated, yet they account for an outsized share of COVID-19 hospitalizations and about half of all intensive care patients.

With hospitals postponing surgeries and some 20000 health workers sick with COVID-19, fining those who refuse vaccinations becomes "a question of fairness," Legault said.

The new "health contribution" will apply only to unvaccinated adults without medical exemptions. The government is still working out the details, but Legault said the levy would be "significant" and higher than $\$ 100$.

\section{Do penalties boost vaccination rates?} Some experts argue that increasing penalties for unvaccinated people may further entrench those opposed to vaccination. Others say that governments should focus on building trust in vaccines and offer rewards for getting the shots instead.

"If you're unvaccinated because you don't trust the government or you don't trust the pharmaceutical industry, it's unlikely that [penalties] will have an influence on your decision," explained Ève Dubé, a medical anthropologist working at the Institut national de santé publique du Québec. "When you push too hard on people that have important distrust issues, you run the risk of a backfire effect, making them even more strongly opposed to vaccination."
In such cases, Dubé said, "one-on-one counselling is one of the most effective interventions for vaccine hesitancy."

But the unvaccinated are not a monolith, and financial penalties may win over people who are "complacent or those who don't feel that the risk:benefit ratio is in favour of vaccination," she said.

Research on the effectiveness of vaccine mandates, financial penalties and other negative incentives in the context of COVID-19 is still limited.

However, a 2020 study of European policies on vaccination against measles and pertussis published in Pediatrics found that mandatory vaccination and higher fines for noncompliance were linked with higher vaccine uptake. Mandating vaccination against measles was also linked with a lower incidence of the disease in countries that didn't allow people to opt out for nonmedical reasons.

Reports from Canada and abroad during the pandemic also suggest that making life difficult for the unvaccinated may sway fence-sitters.

Quebec saw bookings for first vaccine doses jump from a daily average of 1500 to 6000 after announcing proof of vaccination requirements to access liquor and cannabis stores. The province also recorded 7000 bookings the day they announced the tax on unvaccinated adults.

Asked about Quebec's proposed tax, Prime Minister Justin Trudeau said that "very strong measures" including workplace vaccine mandates and restrictions on recreation and travel have worked to boost vaccine uptake.

Quebec, British Columbia, and Ontario saw first dose appointments double after those provinces unveiled vaccine passport policies earlier this year.

And since Canada required federal public servants to get vaccinated or go on unpaid leave, almost $99 \%$ have complied with the policy.

In the United States, the White House reported that major companies that made vaccination a condition of employment have seen vaccination rates increase by an average of $20 \%$.

While some companies have opted for "no jab, no job" policies, Delta Air Lines boosted its employee vaccination rate to $90 \%$ after announcing a US\$200 insurance surcharge for workers who remained unvaccinated.

Meanwhile, it's not clear that offering positive incentives such as cash prizes would move people who are still unvaccinated at this point in the pandemic.

In an American study published in JAMA Health Forum, researchers compared vaccination trends in states that ran lotteries to reward people for getting COVID-19 shots versus states that did not. They ultimately found no association between the lotteries and vaccinations.

Similar lotteries in Alberta and Manitoba failed to make much impact.

According to Shawn Harmon, a policy analyst at Dalhousie University's Health Law Institute, incentives must be targeted to the reasons why people are hesitant. Generic incentives aren't going to mean anything for someone who can't get vaccinated for logistical reasons, or who has intergenerational trauma related to healthcare, he explained. "When we think about incentives, we have to think about who will actually be incentivized."

Is it feasible to fine the unvaccinated? Just over $60 \%$ of 1500 Canadians polled said they would support financial penalties for the unvaccinated similar to those proposed by Quebec.

Legault's government may face legal challenges to taxing the unvaccinated on 
the grounds that the policy violates Charter rights. In such cases, it could fall on the government to justify that the infringement is reasonable.

However, Charter challenges may not hold up given that the government isn't physically forcing people to receive vaccines. And so long as the fine doesn't affect access to care, Quebec's proposal doesn't appear to violate the Canada Health Act.

According to a Quebec spokesperson, the unvaccinated "will never not be able to get the health care that they need, because that's not the essence of the federal law."

Ethically, the picture is more complex.

"We don't tell smokers to pay for their lung cancer treatment. We don't send a bill to someone who didn't wear a seatbelt. Once you're in the health care system, you're a patient," said Vardit Ravitsky, a bioethics professor at the University of Montreal. "What bothers me about the health contribution proposed in Quebec is that it takes us one step closer to these dangerous notions."

It may be justifiable to financially penalize people who refuse vaccinations given the public health threat posed by COVID-19. But many experts argue such penalties should be a last resort after other alternatives have been exhausted.

"We're not there yet. Public health authorities have the jurisdiction to make orders and levy fines in relation to breaches of those orders, but this doesn't seem like it's the right approach or the right time to do this sort of thing," said Harmon.
Mandating vaccination may seem like a "quick and seemingly effective" way to ease pressure on the health system, but it doesn't address the thornier challenges of improving health care delivery or boosting numbers of health workers, Harmon noted.

Ravitsky questioned why Quebec hasn't expanded proof of vaccination requirements before imposing fines - a less coercive step that still "makes life very limiting if you're not vaccinated by choice."

Fines may also disproportionately penalize poorer Canadians and those on the margins of society who may face greater barriers to accessing vaccines. "If it's a flat fee rather than related to your income, then it will obviously impact different families in dramatically different ways and end up becoming an unbearable burden on poor families and a negligible issue for rich families," Ravitsky said.

A spokesperson for Quebec's ministry of finance says the government is looking at "how we're going to help the most vulnerable that have a hard time getting vaccinated," alongside the mechanics of implementing the health contribution.

\section{What countries are fining the unvaccinated?}

Quebec's proposal isn't entirely unprecedented. Since the explosion of Omicron infections late last year, several European countries have announced financial penalties for vaccine holdouts.

Austria, which has one of the lowest vaccination rates in Europe at $68 \%$, was the first Western democracy to announce it would mandate the shots for everyone over 18. Those who don't comply will face fines up to $€ 3600$ every three months.

Germany's new government has since said it will follow Austria's lead, although it has not yet tabled legislation.

In Greece, where more than half a million people over 60 remain unvaccinated, the government will fine seniors $€ 100$ every month if they don't get the shots.

Italy will impose a similar $€ 100$ fee for unvaccinated people over 50 who don't work, while those who have jobs risk fines up to $€ 1500$.

Beyond Europe, the United States and Russia have imposed financial penalties on businesses that fail to mandate vaccinations for their workers. Canada may also fine employers who don't enforce vaccination mandates for federally regulated workers.

Meanwhile, Singapore no longer covers the cost of COVID-19 treatment for patients who are unvaccinated by choice. Those who require intensive care and therapeutics can now expect to pay roughly CA $\$ 23000$ out of pocket, although they may apply for subsidies or tap a national medical savings scheme to partially cover the bill.

\section{Lauren Vogel and Diana Duong, CMAJ}

Content licence: This is an Open Access article distributed in accordance with the terms of the Creative Commons Attribution (CC BY-NC-ND 4.0) licence, which permits use, distribution and reproduction in any medium, provided that the original publication is properly cited, the use is noncommercial (i.e., research or educational use), and no modifications or adaptations are made. See: https://creativecommons.org/ licenses/by-nc-nd/4.0/ 